\title{
Magnetic resonance imaging of spinal cord lesions in multiple sclerosis
}

\author{
LAWRENCE S HONIG, ${ }^{*} \dagger$ WILLIAM A SHEREMATA $\dagger$ \\ From the Department of Neurology, ${ }^{*}$ University of Miami School of Medicine, Miami, FL; and the Department of \\ Neurology, $\uparrow$ Stanford University Medical Center, Stanford, CA, USA
}

SUMMARY The clinical and pathological manifestations of multiple sclerosis are due to areas of demyelination which occur throughout the white matter of the central nervous system. MRI of the brain frequently shows abnormalities in the hemispheric subcortical white matter; these are demonstrable in the majority of patients and support the clinical diagnosis of multiple sclerosis. Our studies have shown that while MRI identifies such cerebral lesions in nearly all clinically definite multiple sclerosis patients with illness of duration greater than 10 years, these areas of abnormal $\mathrm{T}_{2}$ signal are present less often in the brains of patients studied within 3 years of disease onset. However, symptoms referable to the long tracts of the spinal cord are prominent in many of these patients. Imaging of the spinal cord has presented technical problems because of the small size of the cord, patient body, heart and respiratory movements, and limitations of surface coil technology. The spinal cord of 77 patients with multiple sclerosis have been imaged, revealing three types of abnormalities: (1) approximately half the cords show regions of abnormal $T_{2}$ weighted signal; (2) during acute exacerbation, spinal cord enlargement (swelling) may be observed; (3) spinal cord atrophy (narrowing) is found particularly in patients with disease of longer duration and greater disability. Unlike the presence of brain lesions, the existence of spinal cord lesions of high $T_{2}$ signal is not associated with increasing duration of disease but is correlated with disability status. Of patients with such lesions about one fifth did not exhibit brain lesions discernible by MRI.

Multiple sclerosis is diagnosed by use of clinical criteria, ${ }^{\prime}$ although neuro-imaging procedures are increasingly used as paraclinical confirmation of lesions. ${ }^{2}$ Magnetic resonance imaging (MRI) of the brain is considerably more sensitive than X-ray computed tomography, even with "double-dose" intravenous contrast, and demonstrates regions of abnormal $\mathrm{T}_{2}$ signal in the cerebral white matter of ultimately more than $90 \%$ of patients. ${ }^{3-12}$ However, early in the course of disease a smaller proportion of patients show findings on brain MRI..$^{711314}$

Prevalent presenting clinical manifestations of multiple sclerosis consist of motor and sensory long tract signs in the limbs. ${ }^{15}$ These primarily reflect spinal cord disease. For diagnostic purposes, spinal cord MRI obviates need for X-ray myelography to exclude compressive lesions in the spinal canal,,$^{16-18}$ and offers

Address for reprints requests: Lawrence S Honig, PhD, MD, Department of Neurology (C-338), Stanford University Medical Center, Stanford, CA 94305-5235 USA.

Received 3 June 1988 and in revised form 10 October 1988. Accepted 17 October 1988 visualisation of intraparenchymal pathology. Preliminary portions of this report have been presented in abstract form. ${ }^{18}$

Spinal cord is poorly visualised by X-ray computed tomography. ${ }^{1920}$ Although extramedually pathology can be elucidated with intrathecal metrizamide contrast (CT-myelography), intraparenchymal lesions such as syrinx, neoplasms, or demyelination plaques are not easily demonstrated. Such parenchymal cord lesions have been better visualised by MRI, even using body or head (for cervical spine) radiofrequency coils. ${ }^{21-23}$ Factors diminishing MRI quality include motion artifacts owing to cardiac contractions, respiratory excursions and secondary cerebrospinal fluid (CSF) pulsations. ${ }^{16212425}$ In addition, limited spatial resolution, small volumes of affected tissue, partial volume-averaging, and relatively large amounts of overlying tissue in the torso limiting signal-to-noise ratios are impediments. Improved software and hardware, most particularly surface radiofrequency coils $^{1626}$ used in this study have enabled good anatomical discrimination, with higher contrast ratios and better spatial resolution. Techniques such as 
ECG-linked cardiac gating ${ }^{2 \dot{s}}$ enable still better quality images with greater elimination of degradation from cardiac motion and CSF pulsatile flow. Extradural and meduallary lesions are distinguishable, and atrophy, enlargement, syrinx, tumour, vascular malformations and demyelination can usually be discriminated with use of appropriate imaging protocols. $^{170-2327-32}$

\section{Materials and methods}

Study patients all had clinically definite multiple sclerosis by the Schumacher criteria.' The 77 individuals were average age 39.6, SD 10.4 yr (median 38, range 23-65), and consisted of 56 females $(74 \%)$ and 21 males; $74(96 \%)$ were white. Median age at onset of symptoms was 31 with average 32, SD 11 yr. Duration of disease at the time of MRI examination ranged from 1 to 23 years (median 7, mean 8.2, SD 6.3 yr). Expanded disability status scale (EDSS) was evaluated for each patient using the Kurtzke scale ${ }^{33}$ extending from 1.0 to 9.0 with 0.5 increments; our median was 3.5 and mean 4.4 , SD 2.7. Some patients had serial MRI scans; unless otherwise specified the most recent MRI image was utilised in this analysis.

Magnetic resonance imaging systems of Diasonics (46 scans), Picker (48 scans), and Siemens ( 9 scans) were operated using superconducting magnets at field strengths of 0.35 tesla (RF 14.9 MHz: Siemens, and Diasonics 0.5T magnet operated at $0.35 \mathrm{~T}$ ) and 0.5 Tesla (RF 21.3 MHz: Picker), and acquisition matrices of $256 \times 256$ pixels. Multislice imaging exploited spin echo sequences with repetition times (TR) ranging from 500 to $2000 \mathrm{~ms}$ and echo times (TE) ranging from 26 to $80 \mathrm{~ms}$. Spinal cord images were generated using surface coils, and in some cases ECG-gated acquisition modes: sagittal slices $5 \mathrm{~mm}$ thick were obtained, sometimes supplemented by 5 or $10 \mathrm{~mm}$ thick slice axial (transverse) images. Brain images were obtained with $T_{2}$ weighted dual spin echo sequences (for example TR $=2000$, $\mathrm{TE}=28 \& 56)$ acquired as $5 \mathrm{~mm}(69 \%$ of patients $)$ or $10 \mathrm{~mm}$ thick slices ( $31 \%$ patients) in the axial (transverse) plane, following sagittal $T_{1}$ weighted scout sections.

Multiple spin-echo sequences were employed providing $T_{1}$ and $T_{2}$ weighted images. Pulse sequences with shorter $T R$ and TE (for example 600/30) yield substantially $T_{1}$ weighted images useful for visualizing spinal cord outline ${ }^{202829}$ and clearly demonstrate cord narrowing or widening. Pulse sequences with longer TR and TE emphasise $T_{2}$ relaxation time and provide greater sensitivity for the high $\mathrm{T}_{2}$ signals of demyelination plaques with their accompanying increased water content. ${ }^{51231}$ Sequences with very long TR and TE values (such as 2000/80) provide little $T_{1}$ information, but nearly exclusively show $T_{2}$ signal. Such scans form "MRI myelogram" images which lack contrast between spinal cord and subarachnoid CSF, thereby visualising the entire thecal $\operatorname{sac}^{2831}$ and any impingements thereupon; intramedullary lesions with high $T_{2}$ signal may still be prominent. Combinations of long TR and short TE parameters (for example 2000/ 30) yield images possessing greater weighting of proton density, as well as of $T_{2}$ relaxation time, and show high signal, contrast cord and CSF well, at some sacrifice of intraparenchymal contrast.

Several simple grading schemes for brain MRI in multiple sclerosis have been devised ${ }^{89}$ although recently a detailed 45

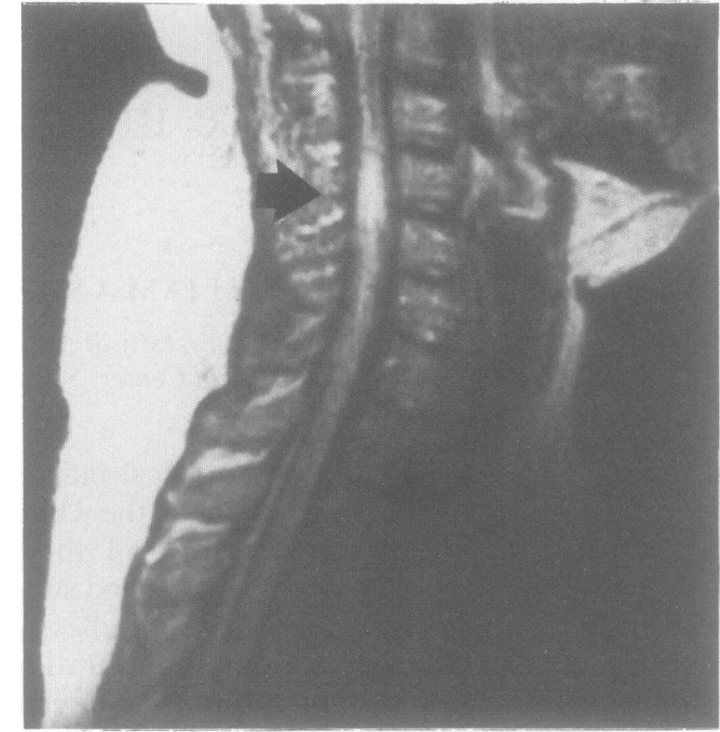

Fig 1 Abnormal MRI signal in the cervical spinal cord. This $44 \mathrm{yr}$ old patient shows an area of cervical cord enlargement and increased $T_{2}$ signal visible at the level of $C 4$ (arrow) in this scan $(T R=1500, T E=80 \mathrm{~ms}$; Picker $)$.

point scale has been proposed. ${ }^{12}$ Our films were graded by the Vanderbilt criteria of Runge $e t$ a $\vec{p}$ according to increasing extent and distribution of lesions on a scale of 0 (normal scan), and 1 to $4 .{ }^{.1}$ The average VGS grade among all brain

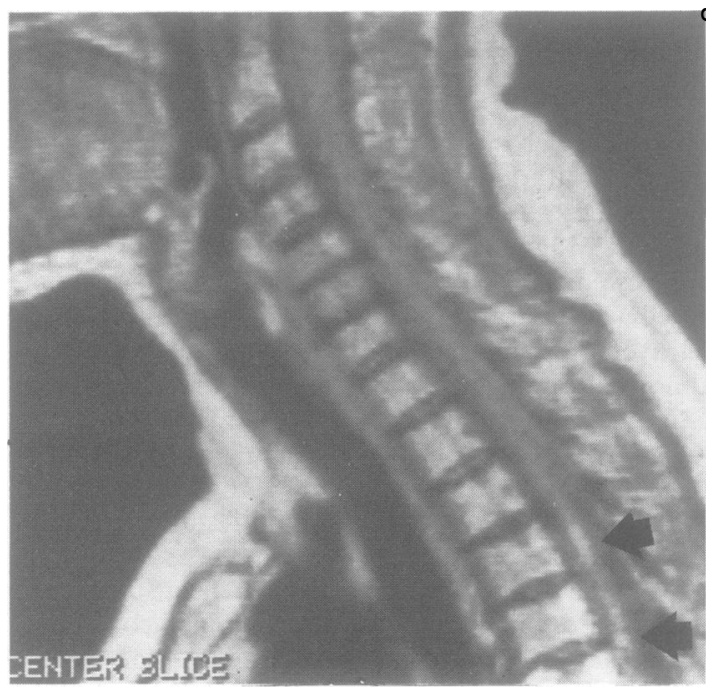

Fig 2 Abnormal MRI signal in the thoracic spinal cord. The patient age 51 yr appears to show two areas (arrows) of increased signal in an otherwise homogeneous spinal cord at vertebral levels $T 4$ and T5/6 in this intermediately weighted image $(T R=2000, T E=28 \mathrm{~ms}$; Diasonics $)$. Small defects in the posterior aspects of vertebral bodies $T 1, T 2$, and T3 represent dural ectasia. 

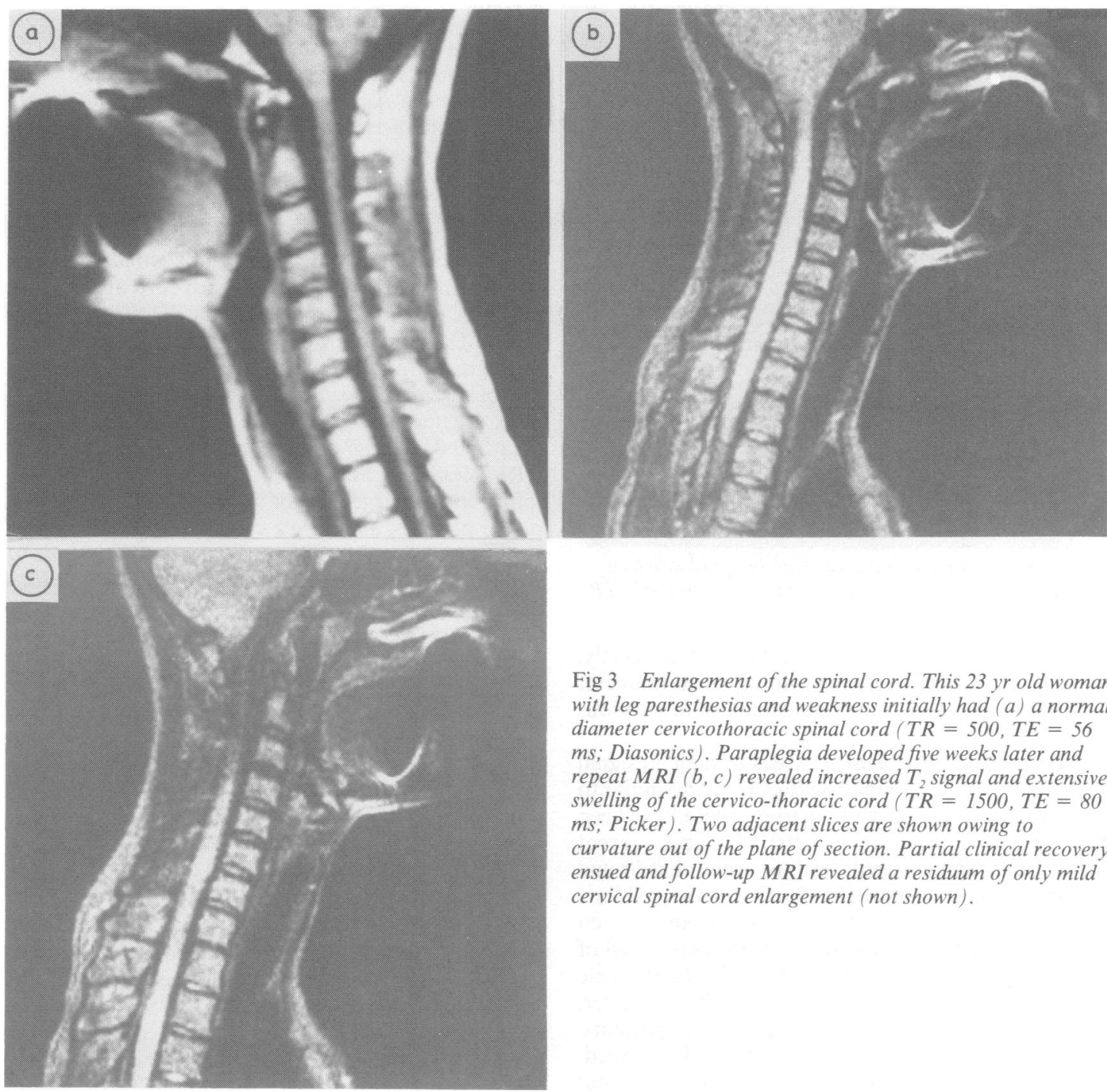

Fig 3 Enlargement of the spinal cord. This $23 \mathrm{yr}$ old woman with leg paresthesias and weakness initially had (a) a normal diameter cervicothoracic spinal cord ( $T R=500, T E=56$ ms; Diasonics). Paraplegia developed five weeks later and repeat $M R I(b, c)$ revealed increased $T_{2}$ signal and extensive swelling of the cervico-thoracic cord $(T R=1500, T E=80$ $m s ;$ Picker). Two adjacent slices are shown owing to curvature out of the plane of section. Partial clinical recovery ensued and follow-up MRI revealed a residuum of only mild cervical spinal cord enlargement (not shown).

scans was 1.96, SD 1.62 (median 2); excluding normal scans, the mean was $2 \cdot 85$, SD $1 \cdot 12$ (median 3 ). All averages are expressed as means plus or minus their standard deviations. Medians and means were almost identical and data had only minimal skew (not significant for EDSS or VGS and p - 0.02 for age and duration). Student's $t$ test was used for statistical comparison of the means of two samples. Statistical analysis of relationships between grouped data was performed by one-way analysis of variance (ANOVA) or Mann-Whitney test (for ordinal data). The $2 \times 2$ contingency tables of data (for example in table 2) were analysed by chi-square test with Yates' correction for continuity. Analyses were accomplished with the aid of the SPSS/PC + microcomputer statistical package.

\section{Results}

Three types of abnormalities were noted in the spinal cord parenchyma of the studied patients with multiple sclerosis: regions of increased $T_{2}$ signal, cord atrophy, and cord enlargement (figs 1-4; table 1). Areas of abnormal signal in these patients' cords behaved like those areas in the brain. Increased signal on intermediate $T_{2}$ weighted images incremented further on second echo acquisitions (that is with more $T_{2}$ weighting). Cord atrophy was described when the diameter appeared abnormally small, and when present usually 


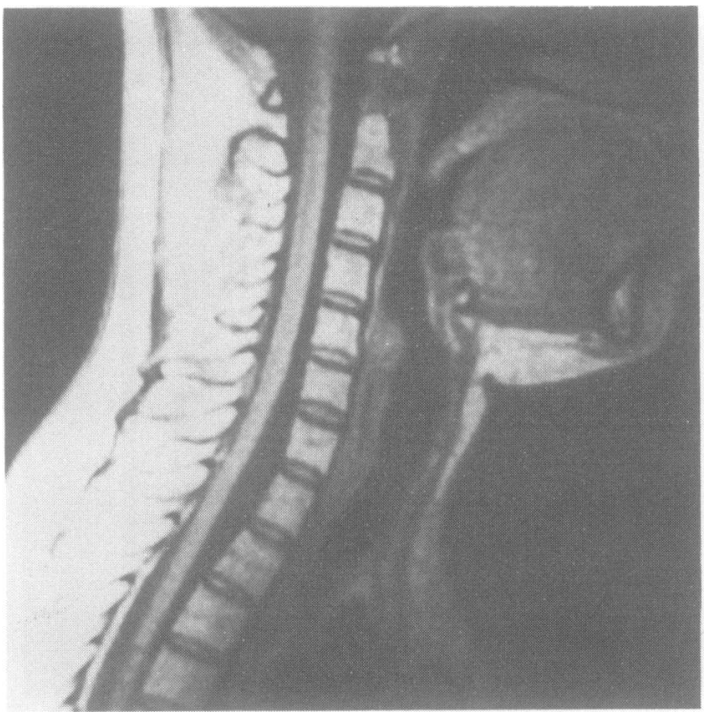

Fig 4 Atrophy of the spinal cord. This 49 yr old with longstanding paraparesis shows atrophy of the thoracic cord (TR $=1000, T E=26 \mathrm{~ms}$; Picker).

represented a large extent of spinal cord. Conversely, enlarged, or swollen cord, was noted when diameter was augmented, relative to the canal, or to previous scans.

Lesions of signal abnormality. Areas of high $\mathrm{T}_{2}$ signal characteristic of demyelination (figs 1-3) are similar to lesions seen in cerebral white matter. These have been observed in isolated prior reports ${ }^{19222331}$ and a recent series. ${ }^{32}$ The spinal cord lesions were distributed throughout the neuraxis (fig 5). Our series exhibits some predilection for cervical cord involvement, even when corrected for the number of scans performed of each region; however, scan sensitivity in the thoracic cord may be lower, due in part to its smaller diameter. High $\mathrm{T}_{2}$ signal was seen in a total of $56 \%$ of patients: $49 \%$ of cervical and $47 \%$ of thoracic cords scanned. Each patient displayed from 0 to 4 lesions, for a total of 63 foci, with a mean of 0.82 lesions/patient, or 1.5

Table 1 Abnormal MRI spinal cord findings

\begin{tabular}{llll}
\hline Spinal cord: & Cervical & Thoracic & Entire \\
\hline Increased T Signal & 38 & 16 & 43 \\
& $(31: 7)$ & $(13: 3)$ & $(34: 9)$ \\
Spinal Cord Atrophy & 13 & 3 & 14 \\
& $(12: 1)$ & $(3: 0)$ & $(13: 1)$ \\
Spinal Cord Swelling & 3 & 1 & 4 \\
& $(1: 2)$ & $(1: 0)$ & $(2: 2)$ \\
Total number scans & 77 & 34 & 77 \\
& $(53: 24)$ & $(24: 10)$ & $(53: 24)$ \\
\hline
\end{tabular}

Every patient received a scan of the cervical spine, but only 34 received thoracic scans. Numbers in parentheses are sublistings of (abnormal:normal) brain findings in each group. lesions/patient among positive scans. Areas of abnormal signal extended from 0.5 to 6 (average $1 \cdot 8$, SD 1.5) vertebral levels (about $1-10 \mathrm{~cm}$ ) longitudinally.

Abnormal cord diameter. Atrophy has been previously reported ${ }^{7}$ and was here noted in $17 \%$ of cervical and $19 \%$ of thoracic cords, involving a total of $18 \%(14 /$ 77) of patients (fig 4). Of the 14 patients with atrophy, five never demonstrated abnormal cord signal, seven were initially accompanied by regions of abnormal cord signal, and two developed abnormal signal on subsequent scans. The patients with cord atrophy did not differ much in age (mean 40.4, SD 9.3 yr) or sex $(10 \mathrm{~F} / 4 \mathrm{M})$ from those without atrophy. However, they did represent a group with significantly longer duration of disease $(12 \cdot 3$, SD 5.3 vs $7 \cdot 3$, SD 6.2 yr; p < $0 \cdot 01$ ), greater disability (EDSS $5 \cdot 9$, SD $2 \cdot 2$ vs $4 \cdot 1$, SD $2 \cdot 7 ; \mathrm{p}=0.03$ ), and more severe brain abnormalities by MRI (VGS 3.1, SD 1.1 vs 1.7, SD 1.6; p < 0.01). Spinal cord enlargement or swelling was only present in four patients, all in acute exacerbation. These three cervical and one thoracic cords all showed lesions of increased $\mathrm{T}_{2}$ signal in the area of cord swelling (figs $1 \& 3)$.

Clinical correlation. The 43 patients showing cord lesions were on the average slightly older $(41 \cdot 3$, SD 11.6) than those without cord lesions (37.6, SD 10.4 yr; $p=0.05$ ), but they did not differ significantly in duration of disease at time of MRI scan (7.6, SD 6.9 vs 8.8 , SD $5.8 \mathrm{yr}$ ). This was in contradistinction to the results of brain imaging in which the $31 \%$ of patients with normal brain images [B-] averaged approximately half as long $(4 \cdot 8$, SD $5 \cdot 2$ yr $)$ duration of disease than those with $[\mathrm{B}+]$ brain lesions $(9 \cdot 8, \mathrm{SD} 6.2 \mathrm{yr})$, a significant difference $(t$ test; $p<0.001)$; ANOVA analysis confirmed a significant $(p=0.001)$ graded relationship with linearity $(p=0.004)$ between duration of disease and actual VGS grade. Despite this difference in duration, the age of $\mathrm{B}+(38 \cdot 0, \mathrm{SD} 11 \cdot 3 \mathrm{yr})$ and B- (40.3, SD $9.9 \mathrm{yr})$ patients differed little. Presence or absence of spinal cord or brain lesions did not correlate with patient sex, ethnicity or age of disease onset.

Increasing disability rated by EDSS score was significant related (ANOVA $p=0.01$ ) to the presence of spinal cord lesions. Even excluding cord atrophy the mean EDSS for patients without spinal disease [S-] was $3 \cdot 50$, SD $2 \cdot 22$ while those with spinal cord lesions [S+] was 5.09, SD $2 \cdot 80$. Within these groups of patients there was an additional weak second order correlation of EDSS and VGS in the group without spinal lesions, but no such relation among those patients with spinal disease. Ranking the mean disabilities of patients with or without brain and spinal findings shows: $\mathrm{S}-\mathrm{B}-2 \cdot 59, \mathrm{SD} 1 \cdot 77, \mathrm{~S}-\mathrm{B}+4.03$, SD 2.33, S + B + 4.69, SD 2.73, S + B - 6.61, SD 2.61. Considering disability in the entire study group, there 


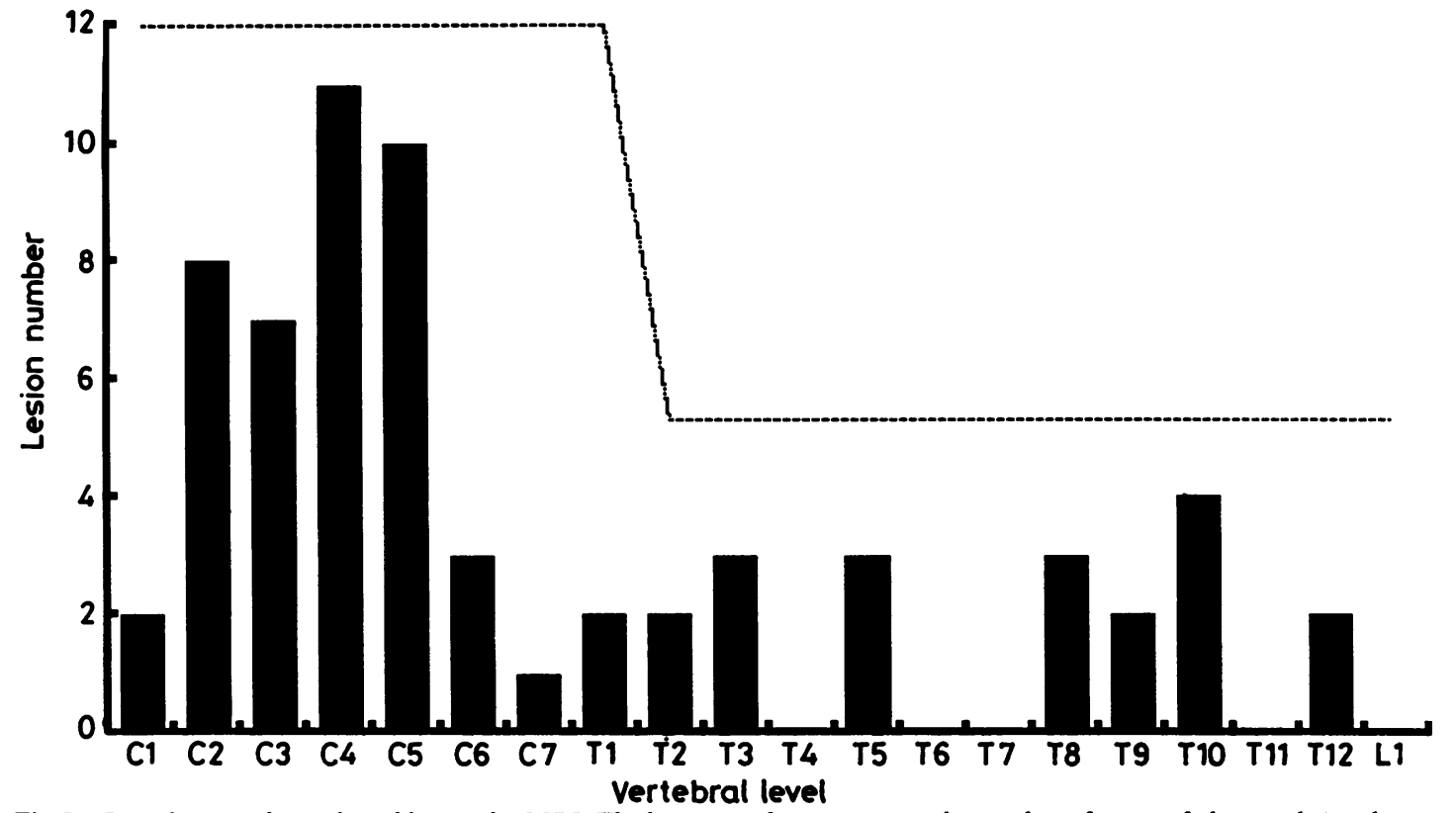

Fig 5 Distribution of spinal cord lesions by MRI. The histogram bars represent the number of areas of abnormal signal at each respective level. The broken line indicates that whereas all 77 patients were imaged in the cervical region, only a subset of 34 were examined in the thoraco-lumbar region. Also note that in any individual, segments of more than one single vertebral level distance were commonly affected (see text). For this histogram, lesions appearing opposite a pair of vertebrae were assigned to the more cephalad level and large lesions were mapped to their middlemost vertebral level.

was no significant relation between EDSS and VGS (ANOVA p > 0.1); B + and B - patients had mean EDSS 4.45 , SD 2.60 and 4.40 , SD 2.98 respectively. Thus EDSS had no significant relation to MRI brain findings, nor did it correlate with age, sex, ethnicity, or duration of disease, although there was a strong relationship to findings of abnormal signal on spinal cord MRI.

Relation of spinal cord and brain findings. Patients with areas of demyelination on brain MRI were significantly more likely to show plaques in their spinal cord $\left(\chi^{2}\right.$ $p=0.05$; table 2). There also was some evidence of a subpopulation with severe brain disease without spinal cord findings. Overall, there was a trend (table

Table 2 Correlation of MRI findings in brain and spinal cord

\begin{tabular}{lllll}
\hline \multicolumn{5}{c}{$B R A I N$} \\
$S$ \\
$P$ & & {$[-]$} & {$[+]$} & TOTAL \\
$I$ & & 15 & 19 & 34 \\
$N$ & {$[-]$} & $(19 \%)$ & $(25 \%)$ & $(44 \%)$ \\
$A$ & & 9 & 34 & 43 \\
$L$ & & $(12 \%)$ & $(44 \%)$ & $(56 \%)$ \\
$C$ & $I+]$ & 24 & 53 & 77 \\
$O$ & & $(31 \%)$ & $(69 \%)$ & $(100 \%)$ \\
$R$ & TOTAL & & \\
$D$ & & &
\end{tabular}

[-] normal or [+] abnormal spinal cord signal.
3) for those having cord lesions to have more extensive brain disease with higher VGS grade (Mann-Whitney $p=0.05$ ). However $38 \%$ of patients without evidence of lesions on brain MRI nonetheless had lesions in the spine.

\section{Discussion}

MRI is now widely used for imaging the cerebral

Table 3 Percentage of spinal cord lesions by brain MRI grade

\begin{tabular}{llll}
\hline $\begin{array}{l}\text { MRI } \\
\text { grade }\end{array}$ & $\begin{array}{l}\text { Cervical } \\
\text { spine }\end{array}$ & $\begin{array}{l}\text { Thoracic } \\
\text { spine }\end{array}$ & $\begin{array}{l}\text { Entire } \\
\text { spine }\end{array}$ \\
\hline 0 & $29 \%$ & $30 \%$ & $38 \%$ \\
& $(7 / 24)$ & $(3 / 10)$ & $(9 / 24)$ \\
1 & $33 \%$ & $33 \%$ & $44 \%$ \\
2 & $(3 / 9)$ & $(1 / 3)$ & $(4 / 9)$ \\
& $80 \%$ & $75 \%$ & $80 \%$ \\
3 & $(8 / 10)$ & $(3 / 4)$ & $(8 / 10)$ \\
& $71 \% \%$ & $50 \%$ & $79 \%$ \\
4 & $(10 / 14)$ & $(3 / 6)$ & $(11 / 14)$ \\
& $50 \%$ & $55 \%$ & $55 \%$ \\
Total & $(10 / 20)$ & $(6 / 11)$ & $(11 / 20)$ \\
& $49 \%$ & $47 \%$ & $56 \%$ \\
& $(38 / 77)$ & $(16 / 34)$ & $(43 / 77)$ \\
\hline
\end{tabular}

MRI Grade is Vanderbilt Grading Scale (VGS) of Runge et al..$^{5}$ Parenthetical expressions $(+/ N)$ indicate in the numerator the number of patients with spinal cords showing lesions $(+)$ and in the denominator the number of patients with that VGS Grade having that cord segment scanned $(\mathrm{N})$. 
plaques of demyelination in multiple sclerosis. It has a much higher sensitivity than X-ray computed tomography, even when performed with double-dose intravenous contrast. ${ }^{4614}$ Without the characteristic periventricular white matter lesions exhibiting increased $T_{2}$ signal, differential diagnosis must be extended to encompass more than demyelinating disease. Nonetheless, of our total group of 77 patients that met the Schumacher' criteria for multiple sclerosis, only $69 \%$ had brain lesions on MRI. These results are in the same range as our previous studies on other patients ${ }^{711}$ although representing a somewhat lower figure than that found by some. ${ }^{361314}$ While we cannot exclude decreased scan sensitivity, more likely explanations include lack of special patient selection and characteristics of our patient referral population in South Florida. When the subgroup of patients with disease of longer duration were selected, there was a higher proportion of abnormal brain results $(78 \% \geqslant 3$ $\mathrm{yr} ; 92 \% \geqslant 12 \mathrm{yr}$ ) in agreement with prior reports. ${ }^{781112}$ In patients with normal brain MRI, cerebrospinal fluid analysis and evoked potentials are often of use is clinical diagnosis." This study shows that the use of spinal cord MRI, especially of the cervical region, may provide anatomical evidence of CNS abnormality. This is particularly important in the group of patients with normal brain MRI. One third of those patients without cerebral findings had abnormal signal in the spinal cord parenchyma.

The abnormal areas of increased $T_{2}$ weighted signal seen in the MRI studies presented cannot be interpreted with certainty to represent regions of active demyelination or gliosis. As with other radiological findings, the pictured abnormalities could arise from a variety of aetiologies. In the CNS, increased transverse proton relaxation time $\left(T_{2}\right)$ usually represents increased tissue water content, which can reflect infarction, neoplastic or infectious processes, as well as inflammatory disease. The anatomical localisation, lesion shape, and contours, generated with different pulse sequences, as well as the clinical context and time-course, aid in radiological diagnosis. The abnormalities in the spinal cord usually involved discrete areas, often multifocal, and were entirely intra-parenchymal without evidence of extradural disease, consistent with findings of other investigators. ${ }^{23} 3132$ Nevertheless, there are likely to be occasional cases, particularly those with spinal cord swelling or enlargement where intrinsic spinal cord tumour cannot initially be excluded. Since the risks of biopsy of the spinal cord are too high, X-ray CT, CSF analysis, and serial, longitudinal follow-up may provide greater diagnostic accuracy.

Much of the presenting and disabling symptomatology of multiple sclerosis corresponds to lesions usually associated with the spinal cord. ${ }^{15}$ For such patients, a significant percentage $(56 \%)$ in our study had abnormalities on MRI of their spinal cord. Distributed throughout the neuraxis, these predominated in the cervical cord. This proportion and skewed topographic distribution is similar to that in another recent published series. ${ }^{32}$ The uneven cervical share may be in part an artifactual consequence of decreased detection sensitivity in the thoracic region, or may reflect true cervical preponderance. The MRI findings show that it is possible to visualise not only cerebral demyelination but also the spinal cord plaques, wellknown to pathologists, ${ }^{153435}$ but previously rarely discernible in the living individual. There is a subpopulation of patients, seen here and previously demonstrated in necropsy studies, in which there are spinal lesions (often together with optic nerve disease: Devic's syndrome) without established brain lesions. ${ }^{1534} 35$

Disability in multiple sclerosis is most often related to "long-tract" disease with its accompanying sensory, balance, and motor deficits. The subcortical cerebral white matter lesions thought by some to be mostly asymptomatic, do tend to increase in number and extent with increasing duration of disease, and may be related to cognitive deficits. ${ }^{36}$ Brainstem lesions are reflected in symptoms such as diplopia, vertigo, and dysarthria, and optic nerve demyelination aso decreased visual acuity, colour desaturation, and scotomata. Kurtzke's disability status scale ${ }^{33}$ reflectso the predominance of long-tract symptomatology with a weighting towards factors such as ambulatory capacity. Our MRI are in consonance with these observations. This study extends our prior results ${ }^{11}$ indicating lack of substantial relationship between EDSS and MRI demonstrable disease of the brain. These results, however, complement antecedent studies by illustrating a significant relationship between EDSS and spinal disease manifest by MRI. Other studies (in preparation and ref 36) suggest there may be a relationship between cognitive and attentional deficits (rather than EDSS score) and cerebral white matter disease seen on MRI. And when the subpopulation of patients without spinal cord disease was alone considered, a weak trend for increasing disability (EDSS) with increasing cerebral MRI disease (VGS) remained: cerebral lesions do have a limited effect on disability. In this paper, duration of disease is once again disclosed as a significant correlate of MRI evinced brain disease. There was no relation of disease duration to spinal cord findings. In part, this likely reflects the prevalence of spinal cord symptoms as presenting traits in multiple sclerosis. ${ }^{\text {is }}$

Current limitations of MRI are due in part to physical factors precluding the imaging of patients with cardiac pacers, ferromagnetic aneurysm clips, metal prostheses, or other foreign bodies in critical 
locations, which might be subject to undesired movement, or heating effects, in addition to causing image degradation. Other shortcomings are caused mostly by movement artifacts secondary to cardiac and respiratory motions, and consequent CSF pulsations, in addition to the relatively long time periods (about $20 \mathrm{~min}$ ) of motionlessness required for generation of a set of images. Improved technology using ECG- and respiratory gating, ${ }^{25}$ advanced pulse sequences, and thinner slice sections (decreasing volume averaging) increase sensitivity, resolution, and discriminatory power. Intravenous contrast agents such as gadolinium-DTPA ${ }^{37-39}$ may allow detection of breakdown of the blood-brain barrier. This may improve detection of the plaques of multiple sclerosis, particularly during acute inflammation ${ }^{39}$ where contrast CT has heretofore been of some use, and allow better assessment of disease activity. Ultimately, use of proton chemical shift information through MRI-spectroscopy, and possibly use of other spin nuclei, may provide increased information.

About half of our study patients had lesions demonstrable in both spinal cord and brain. A significant number $(19 \%)$ showed normal studies of the entire CNS: presumably with plaques in brain or cord not revealed with current technology. A quarter of our group had brain lesions without discernible spinal cord abnormalities. But conversely, $12 \%$ of patients showed normal brain scans but lesions in their spinal cords. Despite limitations, MRI is helpful in diagnosis, and in delineation of spinal cord disease in patients with multiple sclerosis.

We thank Dr M J D Post and her colleagues in the Department of Radiology of the University of Miami Medical Center/Jackson Memorial Hospital, and the members of the Department of Radiology of Mt Sinai Medical Center (Miami Beach) and the NMR Scan Center (Dr Robert Kagan, Fort Lauderdale), for their cooperation and expertise in clinical imaging. We are also grateful to Alan Sazant and Nicholas Bohn for their enthusiastic and vital technical assistance, enabling this study to be made.

\section{References}

1 Schumacher GA, Beebe G, Kibler RF, et al. Problems of experimental trials of therapy in multiple sclerosis: report by the panel on the evaluation of experimental trials of therapy in multiple sclerosis. Ann NY Acad Sci 1965;122:552-68.

2 Poser CM, Paty DW, Scheinberg L, et al. New diagnostic criteria for multiple sclerosis: guidelines for research protocols. Ann Neurol 1983;13:227-31.

3 Young IR, Hall AS, Pallis CA, Legg NJ, Bydder GM, Steiner RE. Nuclear magnetic resonance imaging of the brain in multiple sclerosis. Lancet 1981;ii:1063-6.

4 Brant-Zawadzki M, Davis PL, Crooks LE, et al. NMR demonstration of cerebral abnormalities: comparison with CT. $A J R$ 1983;140:846-54.
5 Runge VM, Price AC, Kirshner HS, Allen JH, Partain CL, James AE. Magnetic resonance imaging of multiple sclerosis: a study of pulse-technique efficacy. AJR 1984;143:1015-26.

6 Jackson JA, Leake DR, Schneiders NJ, et al. Magnetic resonance imaging in multiple sclerosis: results in 32 cases. AJNR 1985;6:171-6.

7 Sheldon JJ, Siddharthan R, Tobias J, Sheremata WA, Soila K, Viamonte M. Magnetic resonance imaging of multiple sclerosis: comparison with clinical, paraclinical, laboratory and CT examination. AJNR 1985;6:683-90.

8 Edwards MK, Farlow MR, Stevens JC. Multiple sclerosis: MRI and clinical correlation. AJNR 1986;7:595-8.

9 Stevens JC, Farlow MR, Edwards MK, Pao-lo Y. Magnetic resonance imaging: clinical correlation in 64 patients with multiple sclerosis. Arch Neurol 1986;43:1145-8.

10 Giesser BS, Kurtzberg D, Vaughan HG, et al. Trimodal evoked potentials compared with magnetic resonance imaging in the diagnosis of multiple sclerosis. Arch Neurol 1987;44:281-4.

11 Honig LS, Siddharthan R, Sheremata WA, Sheldon JJ, Sazant A Multiple sclerosis: correlation of magnetic resonance imaging with cerebrospinal fluid findings. J Neurol Neurosurg Psychiatry 1988;51:277-80.

12 Ormerod IEC, Miller DH, McDonald WI, et al. The role of NMR imaging in the assessment of multiple sclerosis and isolated neurological lesions: a quantitative study. Brain 1987;110: 1579-616.

13 Gebarski SS, Gabrielsen TO, Gilman S, Knake JE, Larack JT, Aisen AM. The initial diagnosis of multiple sclerosis: clinical impact of magnetic resonance imaging. Ann Neurol 1985; 17:469-74.

14 Jacobs L, Kinkel WR, Polachini I, Kinkel RP. Correlation of nuclear magnetic resonance imaging, computerized tomography, and clinical profiles in multiple sclerosis. Neurology 1986;36:27-34.

15 Matthews WB, Acheson ED, Batchelor JR, Weller RO. McAlpine's Multiple Sclerosis. Edinburgh: Churchill Livingstone, 1985

16 Edelman RR, Shoukimas GM, Stark DD, et al. High-resolution surface-coil imaging of lumbar disk disease. AJR 1985; 144:1123-9.

17 Masaryk TJ, Modic MT, Geisenger MA, et al. Cervical myelopathy: a comparison of magnetic resonance and myelography. J Comp Assist Tomogr 1986;10:184-94.

18 Honig LS, Sheremata WA, Shapiro R, Kagan RL. Spinal cord abnormalities without cerebral lesions: a magnetic resonance imaging study of demyelinating disease. Ann Neurol 1986; 20:152.

19 Dee GJ, Bello JA, Hilal SK. High field, thin section nuclear magnetic resonance imaging of the cervical spine. Cardovasc Intervent Radiol 1986;8:283-91.

20 Norman D, Mills CM, Brant-Zawadzki M, Yeates A, Crooks LE, Kaufman L. Magnetic resonance imaging of the spinal cord and canal: potentials and limitations. AJR 1983;141:1147-52.

21 Han JS, Kaufman B, El Yousef SJ, et al. NMR imaging of the spine. AJR 1983;141:1137-45.

22 Hyman RA, Edwards JH, Vacirca SJ, Stein HL. 0.6T MR imaging of the cervical spine: multislice and multiecho techniques. AJNR 1985;6:229-36.

23 Maravilla KR, Weinreb JC, Suss R, Nunnally RL. Magnetic resonance demonstration of multiple sclerosis plaques in the cervical cord. $A J R$ 1985;144:381-5.

24 Enzmann DR, Rubin JB, DeLaPaz R, Wright A. Cerebrospinal fluid pulsation: benefits and pitfalls in MR imaging. Radiology 1986;161:773-8.

25 Enzmann DR, Rubin JB, Wright A. Use of cerebrospinal fluid gating to improve T2-weighted images. Radiology 1987; 162:763-7.

26 Schenck JF, Foster TH, Henkes JL, et al. High-field surface-coil MR imaging of localized anatomy. AJNR 1985;6:181-6.

27 Kulkarni MV, Burks DD, Price AC, et al. Diagnosis of spinal 
arteriovenous malformation in a pregnant patient by MR imaging. J Comp Assist Tomogr 1985;9:171-3.

28 Modic MT, Hardy RW Jr, Weinstein MA, et al. Nuclear magnetic resonance of the spine: clinical potential and limitation. Neurosurgery 1984;15:583-92.

29 Modic MT, Masaryk T, Paushter D. Magnetic resonance imaging of the spine. Radiology Clin North America 1986;24:229-45.

30 Quencer RM, Sheldon JJ, Post MJD, et al. Magnetic resonance imaging of the chronically injured cervical spinal cord. AJNR 1986;7:457-64.

31 Rossi DR, Charney AS. Magnetic resonance imaging of the spine. Semin Neurol 1986;6:84-93.

32 Miller DH, McDonald WI, Blumhardt LD, et al. Magnetic resonance imaging in isolated noncompressive spinal cord syndromes. Ann Neurol 1987;22:714-23.

33 Kurtzke JF. Rating neurological impairment in multiple sclerosis: an expanded disability status scale (EDSS). Neurology 1983;33:1444-52.

34 Adams RD, Kubik CS. The morbid anatomy of the demyelinative diseases. Am J Med 1952;12:510-46.

35 Ikuta F, Zimmerman HM. Distribution of plaques in seventy autopsy cases of multiple sclerosis in the United States. Neurology 1976;26(Suppl):26-8.

36 Honig LS, Ramsay RE, Sheremata WA, Resillez M, Wong P, Sazant A. Magnetic resonance imaging (MRI), cognitive impairment, and the P300 event-related potential (ERP) in patients with multiple sclerosis. Neurology 1986;36(Suppl 1): 157 .

37 Runge VM, Clanton JA, Lukehart CM, Partain CL, James AE Jr. Paramagnetic agents for contrast-enhanced NMR imaging: a review. AJR 1983;141:1209-15.

38 Bydder GM, Brown J, Niendorf HP, Young IR. Enhancement of cervical intrasponal tumors in MR imaging with intravenous Gadolinium-DTPA. J Comp Assist Tomogr 1985;9:847-51.

39 Gonzalez-Scarano F, Grossman RI, Galetta S, Atlas SW, Silberberg DH. Multiple sclerosis disease activity correlates with gadolinium-enhanced magnetic resonance imaging. Ann Neurol 1987;21:300-6.

\section{Early descriptions of cluster headache}

Priority for description of cluster headache or periodic migrainous neuralgia is a source of polemic. Much depends upon the stringency of criteria applied. Hierons ${ }^{1}$ refers to Thomas Willis (1621-1675) who described a venerable matron who began to suffer every afternoon at 4 o'clock from recurring severe headache.

In 1747 Joannes Christoph Ulricus Oppermann published his little acknowledged Dissertatio Medica Inauguralis, entitled Hemicrania Horologica which may be the first account ${ }^{2}$ of the variant, chronic paroxysmal hemicrania, to which I will refer in a later piece.

Benjamin Hutchinson's description of "neuralgia spasmodica" is often cited as the original account of cluster headache: with attacks every 24 hours, but lasting for several hours. The nature of these attacks is dubious, and some were probably tic douloureux.

Romberg in his classic Manual ${ }^{3}$ records: painful sensations in the eye, confined to one side and excited by rays of light and visual effort ... The pupil is contracted. The eye generally weeps and becomes red. These symptoms occur in paroxysms, of a uniform and irregular character, and isolated or combined with facial neuralgia and hemicrania. Romberg's emphasis on the photophobia ${ }^{4}$ is incongruous with current concepts, but he was clearly observing a condition closely akin to cluster headache.

\section{References}

1 Hierons R. Willis's contribution to clinical medicine and neurology. J Neurol Sci 1967;4:1-6.

2 Oppermann, Joannes Christoph Ulricus. Dissertatio Medica Inauguralis. "Hemicrania Horologica" pro gradu doctoris, 1747.

3 Romberg MH. A manual of nervous diseases in man. trans: Sieveking. London Sydenham Society 1983;1:56.

4 Pearce JMS. The enigma of migrainous neuralgia. Physician India 1986;3:141-50. 